\title{
GRANULAR COMPUTING AND EVOLUTIONARY FUZZY MODELLING FOR MECHANICAL PROPERTIES OF ALLOY STEELS
}

\author{
G. Panoutsos and M. Mahfouf \\ Institute for Microstructural and Mechanical Process Engineering: \\ The University of Sheffield (IMMPETUS) \\ Department of Automatic Control and Systems Engineering \\ Mappin St., Sheffield, S1 3JD, UK \\ Email: g.panoutsos@sheffield.ac.uk,m.mahfouf@sheffield.ac.uk
}

\begin{abstract}
In this paper the development of a model for Mamdani type fuzzy rule-based systems using the new concept of granular computing $(\mathrm{GrC})$ is presented. In this study a $\mathrm{GrC}$ algorithm is used to capture the required information in the form of data granules within a high dimensional complex database. The initial collection of information granules is used as a rule-base for a fuzzy inference system (FIS) which is optimised by utilising an Adaptive Genetic Algorithm (AGA). The proposed methodology is applied to real data relating to the heat treatment of alloy steels. Copyright (C) 2005 IFAC
\end{abstract}

Keywords: Fuzzy systems, model approximation, data reduction, knowledge acquisition, genetic algorithms, machine learning

\section{INTRODUCTION}

Fuzzy models have been used in many applications where system simplicity and transparency are required. There have been many approaches to fuzzy modelling, including: linguistic fuzzy modelling (Sugeno and Yasukawa, 1991), data-based relational modelling (Wang and Mendel, 1992), TagakiSugeno - TSK - type fuzzy systems (Takagi and Sugeno, 1985), neural-networks based approaches (Harris, et. al., 2002) and evolutionary computing based approaches (Bastian, 2000). Most fuzzy modelling efforts concentrate on improving modelling performance while maintaining system transparency. Depending on the particular application one can drive the model towards performance (Neurofuzzy, Evolutionary Computing approach) or towards transparency (TSK, Mamdani). This paper takes advantage of the recent developments in Granular Computing- GrC (Bargiela and Pedrycz, 2003) to be used as a method for discovering knowledge within a database. This knowledge is used in the form of a rule-base of a Mamdani type fuzzy system, the simplest and most transparent type of fuzzy systems. Finally, a parametric optimisation of the fuzzy inference engine is performed by the use of an adaptive genetic algorithm. This paper is organised as follows: The concept of capturing knowledge within complex high dimensional databases is presented in Section 2. The translation of the $\mathrm{GrC}$ information granules into a Mamdani fuzzy rule base is shown in Section 3. In Section 4 the optimisation of the resulting fuzzy structure using an adaptive genetic algorithm is discussed. Finally, Section 5 includes a modelling paradigm based on real heat treatment data of alloy steels, and Section 6 includes concluding remarks on the proposed modelling methodology.

\section{KNOWLEDGE DISCOVERY USING GRANULAR COMPUTING}

Data clustering techniques offer a simple way of finding relationships between data sets and grouping data together. Fuzzy C-Means (FCM), the Mountain method and probabilistic measures in fuzzy clustering are among the many clustering techniques 
that have been used for data clustering in fuzzy systems. The main drawback of these methods is that the quality of the solutions (partitions) depends on the initial values (initial cluster centres - FCM), on estimating the number and location of initial clusters' centres (mountain method) and on several other statistical considerations (such as: probabilistic measures for fuzzy clustering).

Conversely, one of the advantages of GrC lies in the fact that granules are grown-evolved (not created neither estimated) from the actual data and therefore there is a strong link between the final data granulation result and the initial data set. The transparency of the granules (or hyper-boxes in the case of more than two dimensions) inspires the possibility of combining GrC with Fuzzy Systems. The control of the level of information abstraction, geometrical measures, similarity and cardinality measures are also amongst the criteria of information granulation using GrC.

In this paper a modified version of the data granulation algorithm, as presented in (Bargiela and Pedrycz, 2003), is used. This recursive algorithm includes the following steps:

- Find the two most compatible information granules and merge them together as a new information granule containing both original granules.

- Repeat the process of finding the two most compatible granules until a satisfactory data abstraction level is achieved.

The most important concept of the above process is the definition of the compatibility measure. This can be purely geometrical (distance between granules, size of granules, volume of granules), density driven (ratio of cardinality versus granule volume) or similarity driven. In this case the compatibility measure is a function of the distance between the granules and a function of the information density of the newly formed granule.

Even though this process can be accidentally identified as hierarchical clustering there is a major difference; each granule consists of the same objects (sub-granules). In hierarchical clustering new objects are created and the boundaries of the new clusters can be in an area where no data are present. The growth of clusters allows strong linkage between the original data set (transparency) and it allows visual monitoring methods for terminating granulation.

Figure 1 shows an example of two-dimensional $(k=2)$ data granulation at various levels of information granulation. The top plot consists of the raw twodimensional data and the subsequent plots show how information is merged to finally form a set of information granules.

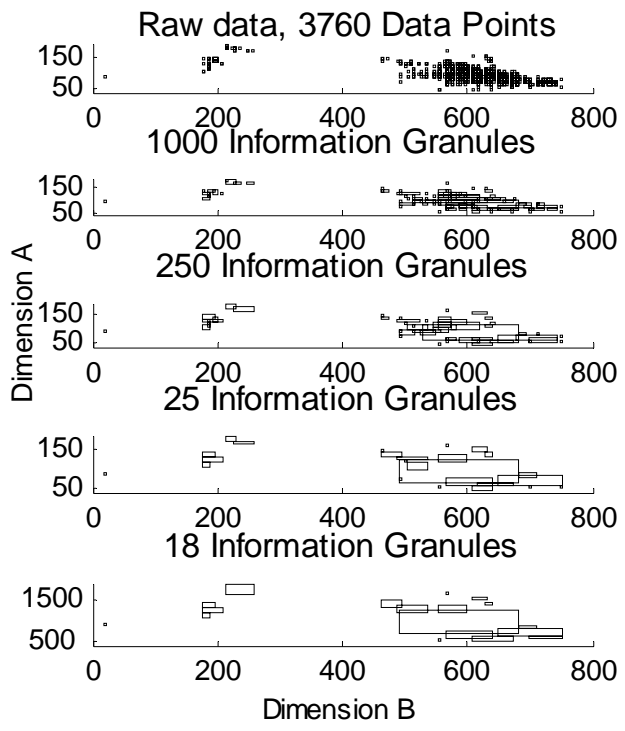

Fig. 1. Snapshot of various levels of data abstraction during an iterative information granulation process.

By considering the merging of each set of granules as some loss of information, it is possible to link the geometrical distance (lost during merging) to the information loss. Information loss can be monitored during the granulation process and this information can be used on-line to decide on a termination point or can also be used off-line in order to determine the granulation performance for various compatibility parameters.

\section{GRANULAR COMPUTING DATA AS A FUZZY RULE BASE}

Consider the granulation of a database provided by a multi-input single-output (MISO) system. By granulating across each input dimension individually and at the same time across the whole input space it is possible to identify relational information (rules) similar to a Mamdani FIS rule-base:

Rule 1:

$$
\begin{aligned}
& \text { if (input } A=A_{1} \text { and input } B=B_{1} \text { and...) } \\
& \text { then }\left(\text { output }=O_{1}\right)
\end{aligned}
$$

Rule 2:

$$
\begin{aligned}
& \text { if (input } A=A_{2} \text { and input } B=B_{2} \text { and...) } \\
& \text { then }\left(\text { output }=O_{2}\right)
\end{aligned}
$$

Where $A_{i}, B_{i}, \cdots, \mathrm{O}_{i}$ are information granules discovered during the GrC process and $i$ : is the number of granules.

The information captured using GrC defines the initial structure of the fuzzy rule-base but the fuzzy system parameters and the inference engine itself is not yet optimised. An evolutionary computing algorithm, such as a Genetic Algorithm, can be used to optimise the system's parameters. 
Even though it is untimely to consider the modelling performance of the FIS at this stage, it is possible to run modelling tests of the raw granules (rule-base) in order to obtain a performance indication, which can be useful during the modelling process. This will be shown in Section 5 using a numerical example.

\section{OPTIMISING THE FUZZY INFERENCE SYSTEM USING AN ADAPTIVE GENETIC ALGORITHM}

Due to the search behaviour and ability of genetic algorithms, they have been used extensively in the past to optimise fuzzy inference systems. In this paper an adaptive genetic algorithm (Srinivas and Patnaik, 1994) will be used to perform parametric optimisation of the FIS, which was obtained using the concept of GrC.

\subsection{The AGA Structure}

The population of this particular GA consists of 100 chromosomes each representing a single FIS (a single model candidate). Each chromosome contains subchromosomes consisting individual granular rules as presented in Section 3. Each granule is represented by a Gaussian membership function of centre $c$ and standard deviation $\sigma$; these parameters will in turn be optimised during the AGA process.

\subsection{Fitness Function}

The fitness function will assess the performance of each individual in the population, and based on Goldberg's linear scaling (Goldberg, 1989), the fittest individuals will evolve. An RMSE fitness function has been selected as follows:

$$
\text { Fitness }=\sqrt{\frac{\sum_{i=1}^{n}(S(i)-D(i))^{T}(S(i)-D(i))}{n}}
$$

Where $n$ : number of training data points, $D(i)$ : model prediction output value for a given $i$-th input data set, $S(i)$ : original output value for a given $i$-th data set.

\subsection{Crossover and mutation}

A single point crossover operation has been selected and the probabilities of crossover and mutation were varied according to the adaptation mechanism. In this particular application (see Section 5 for application details: modelling of properties of alloy steels) the adaptation mechanism has proven to give a superior performance as compared to a simple GA.
The flow diagram of the whole $\mathrm{GrC}$ and AGA modelling process is shown in figure 2 .

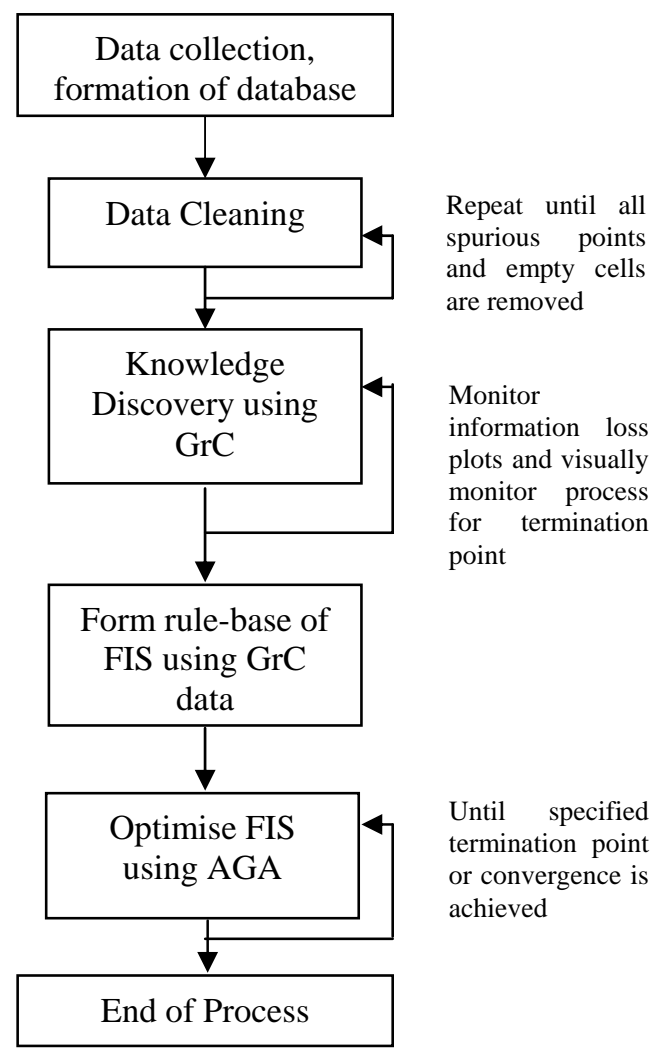

Fig. 2. The Modelling Process Flow Chart.

\section{EXPERIMENTAL STUDIES}

The prediction of properties of heat-treated steel is based on chemical composition and heat treatment data. This is a multiple-input single-output (MISO) process that is difficult to model due to the following reasons: a) non-linear behaviour of the process, b) high interaction between the multi-variable input space, c) uncertainty of the experimental data and d) high complexity of the optimisation space. Black box modelling techniques (such as neural-networks Tenner, 1999) are usually employed to tackle this problem with an acceptable level of performance but fail to make use of experts' (metallurgists) knowledge that will prove to be very valuable. The technique presented in this paper offers a good performance level while maintaining the high transparency of the system during the modelling process. System transparency is often desirable in these kinds of systems as a better understanding of the physical process can be achieved and experts' knowledge can be used along with the model or embedded into the model (incremental learning).

A highly dimensional data set taken from the steel industry is used for modelling purposes. Each set of points represents 15 input variables and 1 output variable. The input variables include both: a) the chemical composition of steel (i.e. \% content of C, $\mathrm{Mn}, \mathrm{Cr}, \mathrm{Ni}$ etc.) and b) the heat treatment data 
(Tempering temperature, Cooling medium etc.). The output variable (the steel property to be modelled predicted) is the Tensile Strength (TS). The TS data set consists of 3760 data points representing steels of various grades. The large TS data set will be used to challenge the ability of GrC to extract and capture information within large and complex databases. A visualisation of the data density in three out of the sixteen possible dimensions is presented in figure 3.
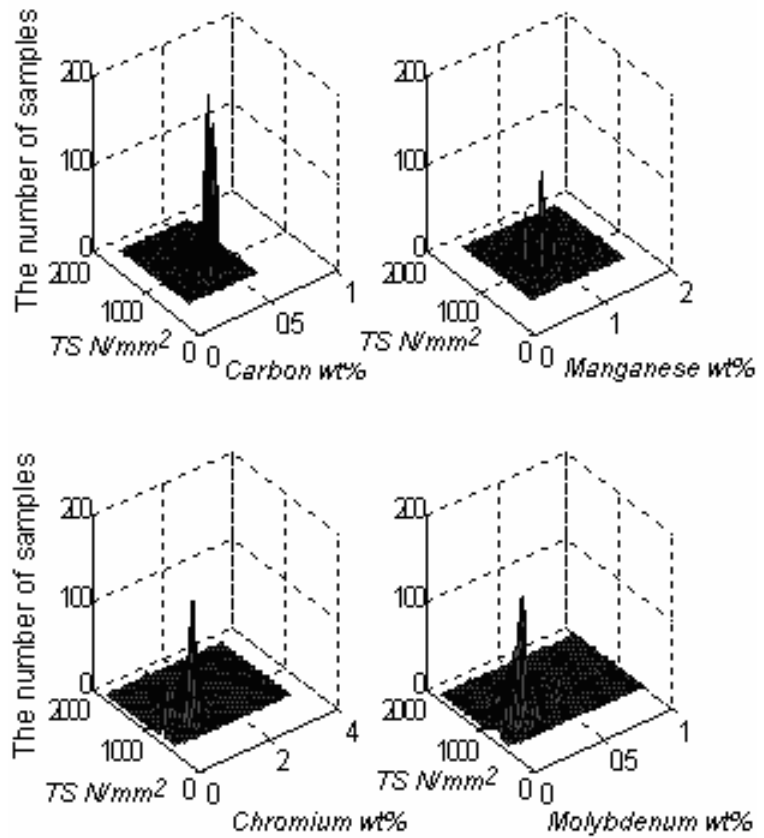

Fig. 3. TS Data density histogram.

The data distribution and density is complex and not homogenous which represents a difficult task for the GrC algorithm to capture knowledge effectively within the sixteen dimensional space.

\subsection{Performance evaluation of information granulation}

Modelling the non-optimised FIS can assess the initial performance of the information granulation process. Using the same system structure as presented in Section 3 a number of FISs are formed using various levels of information granulation data (various number of rules-information granules). In this case the TS data set is used for the modelling process; $75 \%$ of the data are used for the training (information granulation) and the rest is used for the validation of the extracted information granulation model.

The following table presents the performance (Root Mean Square Error - RMSE) of the non-optimised FIS-GrC models, for various levels (number of rules) of information granulation.
Table 1 - Performance of non-optimised FIS-GrC using various levels of granulation, TS Data

\begin{tabular}{ccc}
\hline $\begin{array}{c}\text { No. of rules- } \\
\text { Information } \\
\text { granules }\end{array}$ & $\begin{array}{c}\text { RMSE } \\
\text { Training }\end{array}$ & $\begin{array}{c}\text { RMSE } \\
\text { Validation }\end{array}$ \\
\hline 25 & 104 & 120 \\
50 & 83 & 105 \\
100 & 58 & 92 \\
150 & 37 & 82 \\
\hline
\end{tabular}

As expected (from the theory) the higher the number of information granules the better the performance of the system. The drawback is that the transparency level and maintainability of the FIS-GrC system is reduced as the number of granules is increased. The imbalance between the training and validation performance, which can be seen in Table 1 , was expected because the fuzzy structure is not yet optimised. The performance of the validation (generalisation ability) will be dramatically improved by optimising the fuzzy inference engine, as will be shown in the next section.

\subsection{Post-Optimisation results of FIS-GrC model}

Using past experience on the paradigms relating to the Mamdani FIS, it has been decided that 50 rules (information granules) can achieve a good accuracy level while preserving the high transparency and maintainability of the system. Therefore, the objective of the optimisation algorithm is to maintain the training performance previously seen with 150 granules (Table 1) while reducing the number of granules to 50 and improving the validation performance. The optimisation structure discussed in Section 4 is used.

Figure 4 shows the progress of the objective function within the AGA optimisation. The performance of the best individual is shown in table 2 .

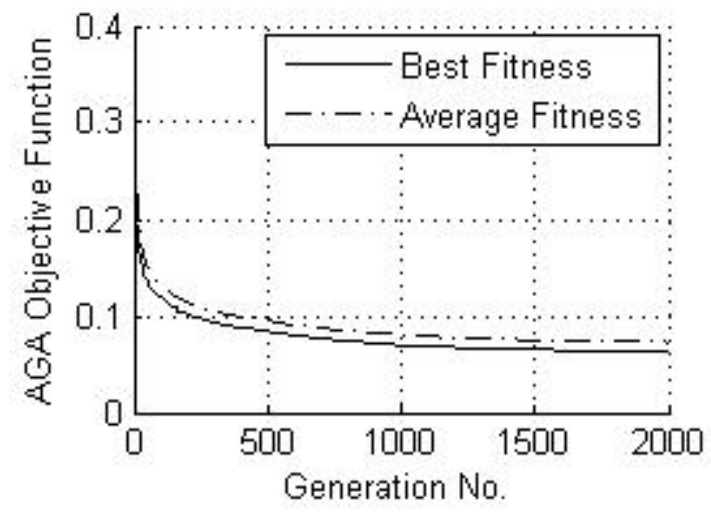

Fig. 4. AGA Objective Function. 
Table 2 - Performance of optimised FIS-GrC, TS data

\begin{tabular}{cccc}
\hline $\begin{array}{c}\text { No. of } \\
\text { rules-Inf. } \\
\text { granules }\end{array}$ & $\begin{array}{c}\text { RMSE } \\
\text { Training }\end{array}$ & $\begin{array}{c}\text { RMSE } \\
\text { Validation }\end{array}$ & $\begin{array}{c}\text { RMSE } \\
\text { Combined }\end{array}$ \\
\hline 50 & 41.34 & 53.96 & 48.07 \\
\hline
\end{tabular}

A plot of the modelling results, measured versus predicted values (TS), are presented in figure 5 .
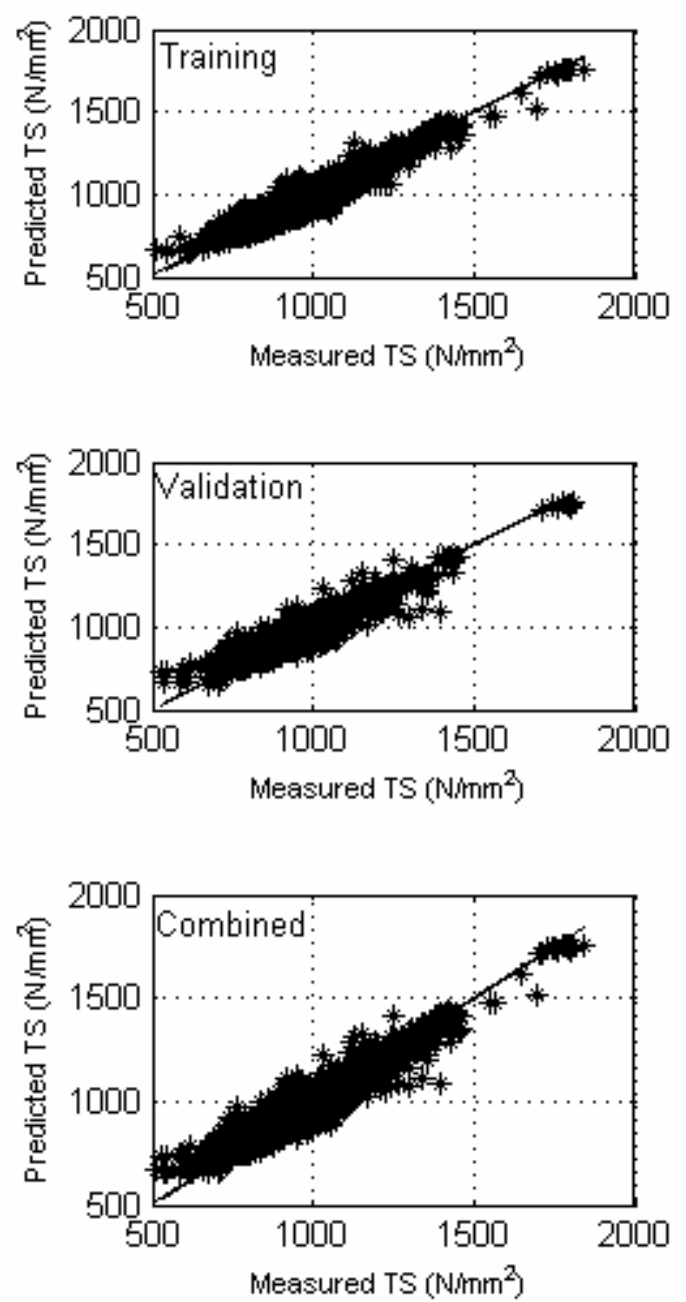

Fig. 5. GrC-FIS, Training, Validation, Combined Performance, of the TS data set.

Training and validation performance is comparable to NN, NF-Mamdani and NF-TSK performance levels. The introduction of 'noise' during validation is expected as dome unseen data points are not included in the rules' structure.

A visualisation of two of the optimised Mamdani fuzzy rules is shown in figure 6. Each variable is shown individually (only 6 out o 16 are shown and only two rules instead of fifty for simplicity). The rule structure presented is the one described by equation 4.

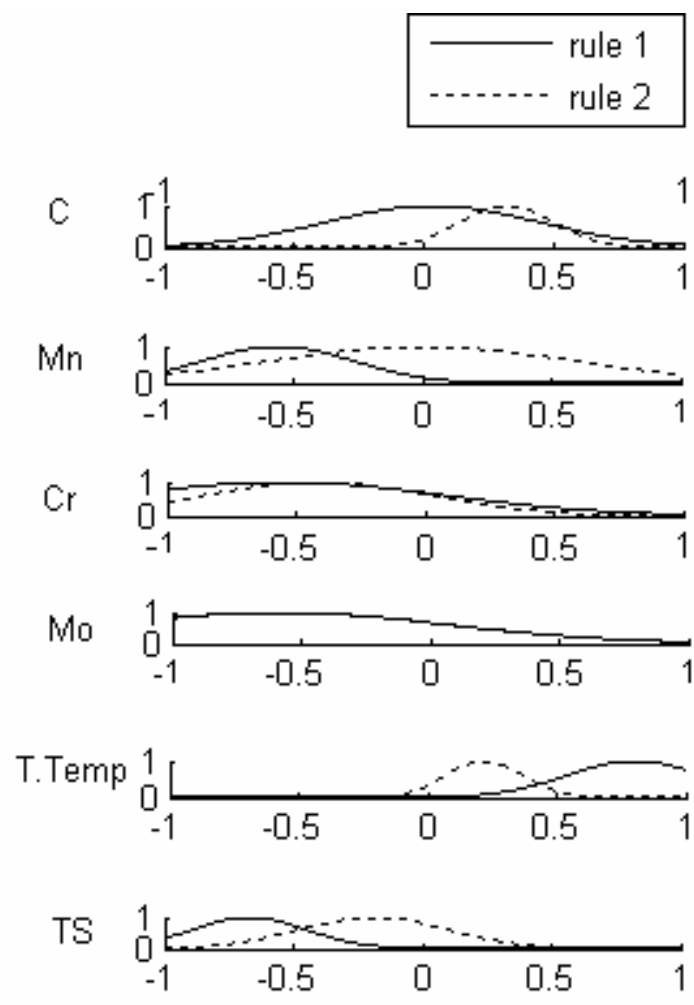

Fig. 6. Mamdani type fuzzy rules of the optimised (GA) system.

As can be seen from figure 7 , the input variables include chemical compositions as well we heat treatment data coded into fuzzy sets. Heat treatment data include test depth and size of the sample taken, test site were the alloy was produced, hardening and tempering temperature and cooling medium. The transparency of the system can be verified by the linguistic interpretability of the rules, i.e. using figure 7: Rule1: "High T.Temp $\rightarrow$ low TS" and by observing Rule 2: "Lowering T.Temp $\rightarrow$ TS is increased". This modelled behaviour is also confirmed by theory and expert's (metallurgist) knowledge.

\subsection{Performance comparison}

By comparing the FIS-GrC modelling technique to current black-box modelling techniques (NN, NF) it is possible to see the similarity in performance level between all methodologies for the given paradigm.

For instance, a NN approach has also been investigated for the paradigm presented in this section. An unseen data set, consisting of twelve new data points has been used for comparison. The performance of the two methodologies can be seen in Table 3 and Figure 7. 
Table 3 - Performance of FIS-GrC as compared with a NN approach, new TS data (12 data points)

\begin{tabular}{ccc}
\hline $\begin{array}{c}\text { Measured } \\
\text { TS }\end{array}$ & $\begin{array}{c}\text { NN } \\
\text { Predicted } \\
\text { TS }\end{array}$ & $\begin{array}{c}\text { GrC-FIS } \\
\text { Predicted } \\
\text { TS }\end{array}$ \\
\hline $\mathbf{1 3 1 9}$ & 1268 & 1302 \\
$\mathbf{1 3 5 4}$ & 1271 & 1336 \\
$\mathbf{9 7 0}$ & 985 & 1015 \\
$\mathbf{1 0 3 8}$ & 982 & 1005 \\
$\mathbf{9 0 8}$ & 1002 & 948 \\
$\mathbf{8 9 4}$ & 945 & 905 \\
$\mathbf{9 1 8}$ & 929 & 942 \\
$\mathbf{9 0 9}$ & 930 & 949 \\
$\mathbf{9 5 6}$ & 930 & 949 \\
$\mathbf{7 4 0}$ & 734 & 852 \\
$\mathbf{7 3 7}$ & 734 & 776 \\
$\mathbf{6 8 9}$ & 698 & 756 \\
\hline & RMSE: & RMSE: \\
& 46.23 & 46.73 \\
\hline
\end{tabular}

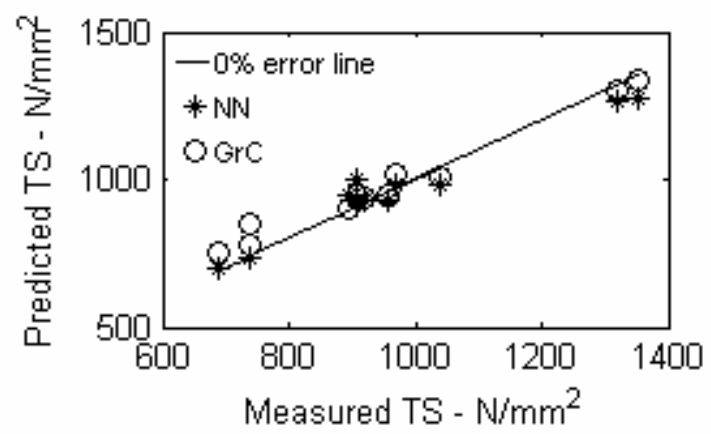

Fig. 7. NN, GrC-FIS fit of new unseen data (12 data points).

The FIS-GrC technique has a comparable performance but not superior as compared to blackbox modelling techniques (based on tests on the same application), as it was expected due to the transparency-performance contradictory nature of the objectives.

On the other hand the combination of GrC with a Mamdani FIS offers transparency levels that are by far superior as compared to black-box or grey-box modelling methodologies.

\section{CONCLUSION}

Fuzzy modelling and knowledge discovery, within databases containing complex relationships, using Granular Computing is discussed in this paper. By taking advantage of the ability of $\mathrm{GrC}$ to capture knowledge in a transparent way and combining this knowledge with a Mamdani fuzzy inference engine it is possible to build a very transparent system capable of modelling various complex processes. As shown in the previous sections the system needed to be optimised via an adaptive genetic algorithm.
The proposed modelling methodology has comparable performance with black-box modelling techniques (Neural Networks, Neural-Fuzzy) but offers very high transparency during the modelling process and the final model is also of high transparency. Due to the transparency of the process, experts' knowledge can be used during the modelling process for deciding various modelling or optimisation parameters, which leads to improved performance of the final model. Additionally, the transparency of GrC will always help to develop in the area of incremental learning, and system adaptation via fuzzy systems. By granulating new data sets using $\mathrm{GrC}$ it is possible to explore the technique of combining new knowledge with the existing rule-base without significant loss of performance.

\section{ACKNOWLEDGMENTS}

The authors would like to thank the Engineering and Physical Sciences Research Council (EPSRC), UK for their financial support under grant GR/R70514/01 and Corus Engineering Steels (CES) Stocksbridge, Sheffield, UK for their help and in providing the heat treatment data.

\section{REFERENCES}

Bargiela, A, Pedrycz W (2003) Granular Computing An Introduction. Kluwer Academic Publishers.

Bastian A (2000). Identifying fuzzy models utilising genetic programming. Fuzzy Sets Syst., 113(3):333-350.

Goldberg DE (1989). Genetic Algorithms in search optimisation and machine learning. Addison Wesley, USA-Canada

Harris C, Hong X, et al. (2002). Adaptive Modelling, Estimation and Fusion from data, A Neurofuzzy Approach. Berlin Heidelberg, New York, Springer-Verlag.

Srinivas M, Patnaik LM (1994). Adaptive Probabilities of Crossover and Mutation in Genetic Algorithms. IEEE Trans. On Systems, Man and Cybernetics 24(4)

Sugeno M, Yasukawa T (1991). Linguistic modelling based on numerical data. Proc. IFSA '91. pp. 264-267

Takagi T, Sugeno M (1985). Fuzzy identification of systems and its application to modelling and control. IEEE Trans. On Systems, Man \& Cybernetics 15: 116-132.

Tenner J (1999). Optimisation of the Heat Treatment of Steel using Neural Networks. Ph.D. Thesis, Department of Automatic Control and Systems Engineering, University of Sheffield, UK.

Wang LX, Mendel JM (1992). Generating fuzzy rules by learning from example. IEEE Trans. On Systems, Man \& Cybernetics 22(6). 